\title{
Cost effectiveness of human papillomavirus test of cure after treatment for cervical intraepithelial neoplasia in England: economic analysis from NHS Sentinel Sites Study
}

Rosa Legood lecturer in decision modelling ${ }^{1}$, Megan Smith program manager, cancer modelling ${ }^{2}$, Jie-Bin Lew senior research programmer ${ }^{2}$, Robert Walker senior research programmer ${ }^{2}$, Sue Moss professor of cancer epidemiology ${ }^{3}$, Henry Kitchener chair of gynaecological oncology ${ }^{4}$, Julietta Patnick director at NHS cancer screening programmes ${ }^{5}$, Karen Canfell associate professor and cancer modelling program leader ${ }^{26}$

\footnotetext{
${ }^{1}$ Health Services Research and Policy Unit, London School of Hygiene and Tropical Medicine, London WC1 9HS, UK; ${ }^{2}$ Cancer Research Division, Cancer Council NSW, Woolloomooloo, NSW 2011, Australia; ${ }^{3}$ Centre for Cancer Prevention, Queen Mary University of London, Wolfson Institute of Preventive Medicine, London EC1M 6BQ; ${ }^{4}$ School of Cancer and Enabling Sciences, University of Manchester, St Mary's Hospital, Manchester M13 9WL, UK; ${ }^{5} \mathrm{NHS}$ Cancer Screening Programme, Sheffield S10 3TH, UK; ${ }^{6}$ School of Public Health, University of Sydney, Australia
}

\begin{abstract}
Objectives To evaluate the cost effectiveness of human papillomavirus testing after treatment for cervical intraepithelial neoplasia (CIN).

Design Economic analysis using a Markov modelling approach to combine cost and epidemiological data from the NHS Sentinel Sites Study with data from previous studies of post-treatment recurrence rates.

Setting English NHS Cervical Cancer Screening Programme.

Interventions Management guidelines after treatment of CIN involving annual cytology follow-up for 10 years, compared with alternative protocols using the human papillomavirus test to reduce the amount of post-treatment surveillance.

Main outcome measures Cases of underlying CIN3+ averted at 10 years and costs per 1000 women treated.

Results Model predictions indicated that, at observed levels of compliance with post-treatment recommendations, management with only cytological follow-up would result in 29 residual cases of recurrent CIN3+ by 10 years and would cost £358 222 (€440 426; \$574 910) (discounted) per 1000 women treated. Implementation of human papillomavirus test of cure in cytologically negative women according to the sentinel sites protocol would avert an additional 8.4 cases of $\mathrm{CIN}$ $3+$ and reduce costs by $£ 9388$ per 1000 women treated.

Conclusions Human papillomavirus test of cure would be more effective and would be cost saving compared with cytology only follow-up. The
\end{abstract}

results of this evaluation support the full scale implementation of human papillomavirus test of cure after treatment of CIN within the NHS Cervical Screening Programme.

\section{Introduction}

Cervical screening in England is recommended every three years for women aged 25-49 years and every five years for women aged 50-65 years, using liquid based cytology. Until recently, guidelines for follow-up after treatment for high grade cervical intraepithelial neoplasia (CIN) recommended annual cytological testing for at least 10 years after treatment; for women treated for low grade disease, cytological follow-up was recommended at six, 12 , and 24 months, and if all results were negative then women were returned to routine recall. ${ }^{1}$ An alternative strategy is to use DNA testing for human papillomavirus as a "test of cure" to discharge women back to routine screening earlier and thus reduce the amount of post-treatment surveillance.

A recent study of human papillomavirus as a test of cure in England involved follow-up at six, 12, and 24 months after treatment and concluded that women who were human papillomavirus and cytology negative at six months could be safely returned to routine recall. ${ }^{2}$ On the basis of these findings, the National Health Service Cervical Screening Programme carried out an implementation project of human papillomavirus 
testing after treatment at six sentinel sites, which returned women to routine recall after a single negative cytology and negative human papillomavirus test result at six months after treatment. $^{3}$ This formed part of a wider study of human papillomavirus triage (the Sentinel Site Study). Human papillomavirus testing in the study involved Hybrid Capture technology (HC2, Gaithersburg, MD, USA), with a cut-off value of 2 relative light units (RLU) used to determine positivity. The study took place at Bristol, Norwich, Liverpool, Manchester, Northwick Park, and Sheffield, which together screen approximately $10 \%$ of women in the English national cervical screening programme.

To date, little evaluation of the cost effectiveness of using human papillomavirus testing in the management of women treated for CIN has taken place. We identified only two published studies: one done in the Netherlands indicated that human papillomavirus testing used for this purpose was a potentially cost effective strategy compared with three repeat cytology tests at six, 12, and 24 months ${ }^{4}$; the second, a US study, found that post-treatment human papillomavirus testing increased costs but added little to no improvement in life expectancy. ${ }^{5}$ The aim of our study was to use data from the Sentinel Site Study to develop a model of post-treatment management and to then use the model to assess the cost effectiveness of different strategies involving human papillomavirus testing compared with cytology only strategies, taking into account realistic levels of expected compliance with follow-up recommendations.

\section{Methods}

We developed a mathematical model using a Markov approach, with a six month time step to evaluate the cost effectiveness of alternative options. The model simulated a cohort of women who had been treated for CIN grades 1, 2, and 3. The simulated cohort was structured in terms of age and grade of treated disease to reflect the cohort included in the Sentinel Sites Study, in which $63 \%$ of treated women were aged under 35 years and $10 \%$ of women were treated for CIN1 and the remainder for $\mathrm{CIN} 2 / 3 .{ }^{3} \mathrm{We}$ included age in the model because many factors are age dependent, including screening recommendations, compliance with screening, mortality from other causes, the probability of hysterectomy for benign disease, the rate of unsatisfactory colposcopy results, and natural history parameters. Further details of the model structure and parameters are available in the web appendix.

We calculated costs and effects for all women over a 10 year time horizon, using a health services perspective. In line with best practice, all costs were discounted at $3.5 \%{ }^{6}$ We chose the time horizon to capture downstream management costs for alternative post-treatment management pathways, as previous recommendations for management of women treated for high grade CIN involved annual cytological follow-up for at least 10 years.

\section{Outcome measures}

We defined the outcomes of interest as underlying cases of CIN3+ averted and total costs (discounted) over 10 years. We did not consider costs related to initial treatment for CIN, as these are equivalent for all strategies considered. We did not calculate quality of life measures (quality adjusted life years), because of the paucity of utility weight data related to post-treatment management for specific time points after treatment.

\section{Management pathways}

We simulated three alternative post-treatment management pathways.

Cytology only follow-up (follow-up based only on cytology) -Women treated for CIN2/3 were assumed to have annual follow-up for 10 years after treatment; those with a cytology result of borderline dyskaryosis or worse at any visit have colposcopy. Women treated for low grade disease have cytology testing at six, 12, and 24 months; if all results are negative, they are returned to routine recall; otherwise, annual follow-up is continued until negative cytology results are obtained on two consecutive occasions.

Sentinel site protocol (post-treatment follow-up incorporating human papillomavirus testing and cytology at six months) - Women for whom cytology is negative at six months have human papillomavirus testing; if this is negative, they are returned to routine recall. Women with borderline dyskaryosis or worse on cytology or who are human papillomavirus positive at six months have colposcopy. Women in whom CIN2+ is detected and treated during the 10 year period are followed up after subsequent treatment with cytology and human papillomavirus testing at six months. Subsequent management is as if this was their first treatment.

\section{Extended human papillomavirus follow-up protocol} (post-treatment follow-up incorporating human papillomavirus testing and cytology at six and 12 months and cytology alone at 24 months) $)^{2}$-Women with negative cytology and human papillomavirus results at both six and 12 months post-treatment have repeat cytology at 24 months post-treatment. If cytology is negative at 24 months, they are returned to routine recall. Women with a cytological result of borderline dyskaryosis or worse or who test human papillomavirus positive at any stage have colposcopy. Women who are treated for CIN2+ during the 10 year period are subsequently followed up with cytology and human papillomavirus testing at six and 12 months and cytology alone at 24 months. All management after subsequent treatment is as if this were their first treatment.

Further details of subsequent management pathways are included in the appendix.

\section{Data sources and assumptions Costs}

We based costs of consumables and capital equipment for human papillomavirus testing on indicative prices from the manufacturer and combined them with observed data on costs of staff time from the Sentinel Sites Study. All other costs came from the literature and are shown in table $1 \Downarrow$. We adjusted costs to 2009 prices by using the Hospital and Community Health Services Index. ${ }^{7}$

\section{Treatment success}

We assumed that either large loop excision of the transformation zone or cone biopsy was used for the treatment of CIN. We assumed either that women treated for CIN were successfully treated (but may or may not remain human papillomavirus positive) or that treatment was unsuccessful (with residual disease, either CIN1 or high grade CIN2+). The proportion in each of these groups was calibrated such that histological detection of CIN at the six month visit was consistent with that found in a previous study and also with observed data from the Sentinel Sites Project (see appendix for details). ${ }^{23}$ We assumed that no fewer than $95 \%$ of women would be successfully treated, in line with guidelines which stipulate that the proportion of 
confirmed treatment failures should not exceed 5\% within 12 months of treatment. ${ }^{1} \mathrm{We}$ also assumed that $16 \%$ of women who have been successfully treated for any grade of CIN remain human papillomavirus positive at six months, consistent with the findings of a previous systematic review. ${ }^{8}$

\section{Risk of recurrent disease after treatment}

We adapted the natural history of the general population at risk and the post-treatment rate of disease recurrence for women treated for high grade CIN from a previously described model. ${ }^{9-12}$ We assumed that women treated for CIN1 were histologically negative for CIN after successful treatment but that such women may still harbour cervical human papillomavirus infection. We assumed the natural history after treatment for CIN1 to be consistent with the general female population with an intact cervix (that is, if a woman in this group was subsequently infected with human papillomavirus, the infection could persist, progress, or clear at rates consistent with the general population at risk; and if a woman was human papillomavirus negative after treatment, the risk of a new infection would be the same as for other women of the same age). ${ }^{9-12}$

We assumed women treated for histologically confirmed CIN $2 / 3$ to be at an increased risk of recurrent CIN2+. We reviewed the evidence to identify the risk of recurrent disease after treatment and assumed that among women developing recurrent high grade disease, $32 \%$ develop CIN2 and 68\% develop CIN $3+{ }^{13-15}$ On the basis of these data, we modelled the risk of each grade of recurrent disease developing and being detected after successful treatment, according to post-treatment human papillomavirus status. ${ }^{2} 8131416-20$ We then calibrated model predictions of CIN2/3 detection rates at 12,24 , and 72 months to published data. ${ }^{217}$ The model predictions, targets based on observed data, and details of parameters used in the natural history model are included in the appendix.

\section{Compliance with follow-up recommendations}

We assumed compliance with the six month post-treatment visit to be $100 \%$, because the purpose of our evaluation was to assess the relative cost effectiveness of different management strategies after this visit. We based compliance rates at 12 and 24 months (which were six and 12 months after the previous visit) on observed data and assumed them to be $85 \%$ and $83 \%$ among women who had attended the previous visit; we based this on compliance data from a key relevant study. ${ }^{2}$ Because we predicted the outcomes following a new intervention in women after treatment, long term observational data on compliance are not available. The observational study followed women for a total of 24 months, but in the absence of other information we assumed that for women attending subsequent visits with a recall time of six or 12 months, the compliance with follow-up over the specified time would be similar to that observed in the study for women recalled at that interval.

For women referred back to routine screening, we used a previous analysis of registry data from Oxfordshire to estimate the cumulative proportion re-screened over time ${ }^{21}$; we used this in conjunction with age specific national coverage data for England to derive an interval specific probability of women in different age groups attending for routine screening, ${ }^{22}$ as previously described. ${ }^{11}$ This allowed us to model the effects of some women having early and late re-screening after being returned to routine screening. Because these data were derived from a region where and at a time when three yearly screening was recommended for all ages, we applied these probabilities only to women with a recommended screening interval of three years (ages 25-49 years). For women with a recommended screening interval of five years (ages 50-64 years), we assumed no early or late re-screening but that all women would re-attend every five years. For women referred to colposcopy, we assumed $84 \%$ compliance, on the basis of national routinely collected data. ${ }^{21}$ In a sensitivity analysis, we also considered a scenario in which compliance with all follow-up visits, colposcopy recommendations, and routine screening was $100 \%$ (that is, perfect compliance).

\section{Test characteristics}

We based estimates of the test accuracy of liquid based cytology on previous meta-analyses, ${ }^{910}$ and we based rates of inadequate cytology on 2007-08 statistics from the cervical screening programme in England. ${ }^{2}{ }^{17} 22 \mathrm{We}$ modelled the performance of HC2 human papillomavirus testing as the probability of a positive test result, given a true underlying health state (defined as normal, human papillomavirus infection, CIN1, CIN2, or CIN3+); these are directly related to the operational sensitivity and specificity of the test. We derived estimates and feasible ranges for $\mathrm{HC} 2$ positivity for each health state from the literature, as previously reported; data sources included summary information from a range of studies for which different $\mathrm{HC} 2$ thresholds were used..$^{11}{ }^{11}{ }^{23}$ We also modelled colposcopy test characteristics as the probability that a biopsy would be taken, given a true underlying health state. Estimates and feasible ranges for these probabilities were based on previous analyses, ${ }^{10}{ }^{11}$ as well as on data from the Sentinel Sites Study (personal communication, Rachel Kelly, Institute of Cancer Research, London).

\section{Sensitivity analysis}

We used a combination of one way and (where appropriate) partial multi-way sensitivity analysis to determine how sensitive the findings of this modelled analysis were to various assumptions. We varied the following parameters over a feasible range of possible values: the age composition of the cohort of women treated for CIN; the proportion of women who were treated for each grade of CIN; the cost of collecting follow-up test samples (reflecting whether this was done in the community or by a specialist in a hospital setting); the test characteristics of HC2 testing, cytology, and colposcopy; and compliance with recommendations.

\section{Results}

Table $2 \Downarrow$ shows the predicted health outcomes, resource use, and costs over 10 years for the alternative post-treatment management strategies considered. We found that cytology only follow-up would result in 29 residual cases of recurrent CIN3+ per 1000 women treated by 10 years. If human papillomavirus testing was implemented according to either of the protocols considered, it would avert approximately eight additional CIN3+ cases per 1000 women treated. In the case of the sentinel sites protocol, most of these additional cases would be averted between four and 10 years after initial treatment (fig $1 \Downarrow$ ).

Cytology only follow-up is estimated to cost $£ 358222$ ( $€ 440$ 426; \$574 910) (discounted) per 1000 women treated over 10 years. The model predicted that post-treatment follow-up with human papillomavirus testing according to the sentinel sites protocol would be cost saving compared with cytology only follow-up (reducing costs by $£ 9388$ per 1000 women treated ), whereas implementation of testing according to the extended human papillomavirus follow-up protocol would be more expensive than cytology only follow-up (by $£ 49052$ per 1000 
women treated). This implies a predicted cost per additional case of CIN3+ averted of $£ 6474$ for the extended human papillomavirus follow-up protocol and a cost saving per additional case averted of $£ 1110$ for the sentinel sites protocol. These differences in costs were mainly driven by differential resource use for each strategy. For the human papillomavirus strategies, more women were initially referred to colposcopy because of the higher overall test positivity associated with adding human papillomavirus testing at the six month visit, but for the sentinel site protocol the total number of colposcopies over 10 years was less than with cytology only follow-up. Although the human papillomavirus strategies were predicted to lead to a higher number of re-treatments, most of these were predicted to occur at the six month follow-up, with fewer subsequent treatments compared with cytology only follow-up (table $2 \Downarrow$ ). The proportion of colposcopies resulting in re-treatment was highest for the sentinel sites protocol (74\%), lowest for cytology only follow-up (53\%), and intermediate for the extended human papillomavirus follow-up protocol (62\%).

\section{Sensitivity analysis}

Figure $2 \Downarrow$ shows the results of a sensitivity analysis for the cost per additional case of underlying CIN3+ averted for each of the human papillomavirus strategies. The findings were most sensitive to assumptions about the assumed level of compliance with follow-up recommendations and the cost of collecting follow-up test samples (that is, whether this was done in the community or by a specialist in a hospital setting); in the case of the sentinel sites protocol, the results were also somewhat sensitive to the assumed test characteristics of $\mathrm{HC} 2$.

Post-treatment management using the sentinel sites protocol became more attractive when the cost of collecting follow-up test samples was assumed to be higher (that is, assuming that they were collected by a specialist in a hospital setting), as human papillomavirus strategies would involve fewer visits overall. However, this strategy became less attractive when HC2 was assumed to be less specific; it also became slightly less attractive when the proportion of the cohort treated for CIN1 was higher, as this group have fewer visits under cytology only follow-up. Assumptions about the test characteristics of cytology and colposcopy had relatively smaller effects.

The overall findings of the evaluation were sensitive to assumptions about compliance with follow-up visits to the extent that they altered the relative ranking of the strategies. If we assumed perfect compliance, we found cytology only follow-up to be comparatively more effective, with fewer cases of underlying CIN3+ at 10 years than for either of the human papillomavirus testing strategies. However, the associated costs were also considerably increased; and although assuming perfect compliance also increased costs for the strategies involving human papillomavirus testing, this occurred to a lesser extent (table $3 \Downarrow$ ). When we compared the relative costs and outcomes of the two human papillomavirus strategies, the extended human papillomavirus follow-up protocol had similar efficacy to the sentinel sites protocol but was associated with higher costs under the scenarios examined in sensitivity analysis.

\section{Discussion}

This evaluation was informed by comprehensive and detailed use of epidemiological and cost data from the NHS Cervical Cancer Screening Programme Sentinel Sites Study and other observational studies, plus data from the screening programme in England. We found that the early use of human papillomavirus testing after treatment averts more cases of
CIN3+ and is also cost saving compared with cytology only follow-up, because it seems to be more effective at the early identification of women who are at risk of recurrent disease in the future. The findings also indicate that the capacity of annual cytology to prevent CIN3+ in women previously treated for CIN depends on a very high level of compliance with annual visits. This is likely to explain, in part, the difference between our findings and those of a previous evaluation, which assumed perfect compliance with follow-up recommendations. ${ }^{5} \mathrm{We}$ also found that human papillomavirus testing according to the sentinel sites protocol results in fewer colposcopies over 10 years than does cytology only follow-up, and a higher proportion of colposcopies resulted in re-treatment. This is potentially an important reduction in the burden of follow-up on women, as well as on the health system. It also indicates that the colposcopies done under this strategy are better targeted at women who will benefit from them. As a result of the successful implementation of the sentinel sites and this cost effectiveness analysis, the human papillomavirus test of cure is now being implemented nationally in England, so the cytology only protocol is no longer current practice.

When we assessed the relative effects and costs of alternative human papillomavirus based strategies, we found that once-only cytology and human papillomavirus testing at six months after treatment is predicted to have comparable effectiveness to a strategy involving three rounds of repeat testing; in addition, the once-only cytology and human papillomavirus based testing strategy was found to be less costly. This finding may be relevant to other settings, especially in countries that have implemented extended human papillomavirus testing protocols. For example, the national screening programmes in Australia and New Zealand have introduced recommendations involving repeated rounds of human papillomavirus and cytology testing after treatment, in which women are required to have negative results in both tests for two consecutive years before they are discharged back to routine screening. ${ }^{24}{ }^{25}$ These recommendations were originally based, in part, on cost minimisation (rather than cost effectiveness) analysis comparing extended human papillomavirus testing with previous recommendations in those settings, which was annual cytological follow-up until the age of 70 . To our knowledge, the comparative cost effectiveness of simplified human papillomavirus test based strategies compared with extended test protocols has not been examined in these settings and is a potential subject for future cost effectiveness analyses.

Follow-up testing at six months post-treatment could also be further streamlined to involve only human papillomavirus testing without cytology. Studies of longitudinal outcomes after primary human papillomavirus testing in the general population show that cumulative rates of $\mathrm{CIN} 3+$ in human papillomavirus negative women are very similar to rates in the group of women who are both human papillomavirus and cytology negative over several years of follow-up, ${ }^{26}$ implying that adjunctive testing with both tests is relatively inefficient compared with using human papillomavirus testing alone. However, further data on the comparative performance of human papillomavirus testing alone versus combined with cytological screening are needed in the post-treatment setting. We were not able to evaluate human papillomavirus testing as a standalone test in this evaluation, because the data used came from studies in which human papillomavirus testing was done at six months only when cytology was negative.

The high negative predictive value of post-treatment management involving human papillomavirus testing and cytology at six, 12, and 24 months has recently been reported 
to be maintained over five years. ${ }^{27}$ Longer term follow-up data from the Sentinel Sites Study will further enable the validity of the observable model predictions (such as rates of colposcopy and re-treatments) to be assessed. Our evaluation of the cost effectiveness of human papillomavirus as a test of cure over a period of 10 years represents a modelled extrapolation of data obtained from studies with shorter follow-up periods. Therefore, although our findings support the implementation of human papillomavirus based strategies for post-treatment management, a need remains for ongoing monitoring and evaluation of the long term safety of these strategies.

\section{Conclusion}

We found that the human papillomavirus test of cure according to the sentinel sites protocol is a more effective and less costly strategy than annual cytological follow-up over 10 years. The findings of this analysis suggest that a single round of cytology and human papillomavirus testing six months after treatment effectively identifies women at future risk of serious recurrent cervical disease. The results support the full scale implementation within the NHS Cervical Cancer Screening Programme of human papillomavirus testing as a test of cure after treatment for CIN.

Contributors: RL, KC, and MS designed the study. MS, RW, J-BL, and $\mathrm{KC}$ implemented the model in TreeAge. $\mathrm{RL}$ and $\mathrm{SM}$ provided epidemiological support and data analysis. RL, KC, MS, and J-BL drafted the initial manuscript. All authors were responsible for redrafting the final manuscript.

Funding: The study was funded by the NHS Cancer Screening Programme. The analyses and decision to publish were independent of the funders.

Competing interests: All authors have completed the Unified Competing Interest form at www.icmje.org/coi_disclosure.pdf (available on request from the corresponding author) and declare: RL, SM, MS, RW, J-BL, and KC were funded by the NHS Cancer Screening Programme to do this work. $\mathrm{KC}$ is involved in configuring a new trial of cervical screening in Australia which will involve support from several manufacturers. Ethical approval: Not needed.

Data sharing: Further details are available in the web technical appendix.

1 Luesley D, Leeson S, eds. Colposcopy and programme management: guidelines for the NHS cervical screening programme. NHS Cancer Screening Programmes, 2004.

2 Kitchener HC, Walker PG, Nelson L, Hadwin R, Patnick J, Anthony GB. HPV testing as an adjunct to cytology follow-up of women treated for cervical intraepithelial neoplasia. BJOG 2008;115:1001-7.

3 Kelly RS, Patnick J, Kitchener HC, Moss SM, for the NHSCSP HPV Special Interest Group. HPV testing as a triage for borderline or mild dyskaryosis on cervical cytology: results from the Sentinel Sites Study. Br J Cancer 2011;105:983-8.

4 Coupe VM, Berkhof J, Verheijen RH, Meijer CJ. Cost-effectiveness of human papillomavirus testing after treatment for cervical intraepithelial neoplasia. BJOG 2007:114:416-24.

5 Melnikow J, Kulasingam S, Slee C, Helms LJ, Kuppermann M, Birch S, et al. Surveillance after treatment for cervical intraepithelial neoplasia: outcomes, costs, and cost-effectiveness. Obstet Gynecol 2010;166:1158-70.

6 National Institute for Health and Clinical Excellence. Guide to methods of health technology appraisal. NICE, 2008

7 Curtis L. Unit costs of health and social care. Personal Social Services Research Unit, University of Kent, 2010
8 Paraskevaidis E, Arbyn M, Sotiriadis A, Diakomanolis E, Martin-Hirsch P, Koliopoulos G, et al. The role of HPV DNA testing in the follow-up period after treatment for CIN: a systematic review of the literature. Cancer Treat Rev 2004;30:205-11.

9 Canfell K, Barnabas R, Beral V, Patnick J. The predicted effect of changes in cervical screening practice. Br J Cancer 2004;91:530-6.

10 Human papillomavirus triage test for women with possible of definite low-grade squamous intrapeithelial lesions. Medical Services Advisory Committee, 2009. (MSAC reference 39).

11 Automation assisted and liquid based cytology for cervical cancer screening. Medical Services Advisory Committee, 2009. (MSAC reference 1122.)

12 Creighton P, Lew J, Clements M, Smith M, Howard K, Dyer S, et al. Cervical cancer screening in Australia: modelled evaluation of the impact of changing the recommended interval from two to three years. BMC Public Health 2010;10:734.

13 Cecchini S, Visioli C, Zappa M, Ciatto S. Recurrence after treatment by loop electrosurgical excision procedure (LEEP) of high-grade cervical intraepithelial neoplasia. Tumori 2002;88:478-80

14 Flannelly G, Langhan H, Jandial L, Mana E, Campbell M, Kitchener H. A study of treatment failures following large loop excision of the transformation zone for the treatment of cervical intraepithelial neoplasia. BJOG 1997;104:718-22.

15 .Rema P, Suchetha S, Thara S, Sebastian P, Wesley MD, Sankaranarayanan R. Effectiveness, acceptability and safety of LEEP in Kerala, India: beginners' experience. 2007. http://screening.iarc.fr/poster/Effectiveness,\%20acceptability\%20and\%20safety\% 20of\%20LEEP\%20in\%20Kerala,\%20India\%20beginners\%20experience.pdf.

16 .Strander B, Andersson-Ellström A, Milsom I, Sparén P. Long term risk of invasive cancer after treatment for cervical intraepithelial neoplasia grade 3: population based cohort study. BMJ 2007;335:1077.

17 Melnikow J, McGahan C, Sawaya GF, Ehlen T, Coldman A. Cervical intraepithelial neoplasia outcomes after treatment: long-term follow-up from the British Columbia Cohort Study. J Natl Cancer Inst 2009;101:721-8.

18 Soutter WP, de Barros Lopes A, Fletcher A, Monaghan JM, Duncan ID, Paraskevaidis $E$, et al. Invasive cervical cancer after conservative therapy for cervical intraepithelial neoplasia. Lancet 1997;349:978-80

19 Soutter WP, Sasieni P, Panoskaltsis T. Long-term risk of invasive cervical cancer after treatment of squamous cervical intraepithelial neoplasia. Int J Cancer 2006;118:2048-55.

20 Chan BK, Melnikow J, Slee CA, Arellanes R, Sawaya GF. Posttreatment human papillomavirus testing for recurrent cervical intraepithelial neoplasia: a systematic review. Am J Obstet Gynecol 2009;200:422.e1-9.

21 Canfell K, Sitas F, Beral V. Cervical cancer in Australia and the United Kingdom: comparison of screening policy and uptake, and cancer incidence and mortality. Med $J$ Aust 2006;185:482-6.

22 Health and Social Care Information Centre. Cervical screening programme, England 2007-2008. Health and Social Care Information Centre, 2008.

23 Arbyn M, Sasieni P, Meijer CJ, Clavel C, Koliopoulos G, Dillner J. Chapter 9: Clinical applications of HPV testing: a summary of meta-analyses. Vaccine 2006;24(suppl 3):S3/78-89.

24 National Screening Unit. Guidelines for cervical screening in New Zealand. National Screening Unit, Ministry of Health, 2008 (available at www.nsu.govt.nz/files/NCSP/NCSP Guidelines_ALL_small(1).pdf).

25 National Cervical Screening Program. Screening to prevent cervical cancer: guidelines for the management of asymptomatic women with screen detected abnormalities. National Health and Medical Research Council, 2005 (available at www.nhmrc.gov.au/files_nhmrc/ publications/attachments/wh39.pdf).

26 Dillner J, Reboli M, Birembaut P, Petry K-U, Szarewki A, Munk C. Long term predictive values of cytology and human papillomavirus testing in cervical cancer screening: joint European cohort study. BMJ 2008;337:a1754.

27 Cruikshank M, Hadwin R, Nelson L, Walker P, Moore C, Kitchener K. HPV test of cure: effective protection for five years. International Papillomavirus Conference, Berlin, Germany, 2011.

28 Moss S, Gray A, Marteau T, Legood R, Henstock E, Masissi E. Evaluation of HPV/LBC cervical screening pilot studies: summary of report to the Department of Health. NHS Cervical Screening Programme, 2004.

29 Martin-Hirsch P, Rash B, Martin A, Standaert B. Management of women with abnormal cervical cytology: treatment patterns and associated costs in England and Wales. Br J Obstet Gynaecol 2007;114:408-15.

30 Sherlaw-Johnson C, Philips Z. An evaluation of liquid-based cytology and human papillomavirus testing within the UK cervical cancer screening programme. Br J Cancer 2004;91:84-91.

Accepted: 06 October 2012

\section{Cite this as: BMJ 2102;345:e7086}

This is an open-access article distributed under the terms of the Creative Commons Attribution Non-commercial License, which permits use, distribution, and reproduction in any medium, provided the original work is properly cited, the use is non commercial and is otherwise in compliance with the license. See: http://creativecommons.org/licenses/by$\mathrm{nc} / 2.0 /$ and http://creativecommons.org/licenses/by-nc/2.0/legalcode. 


\section{What is already known on this topic}

Results of previous studies are inconsistent about whether human papillomavirus (HPV) testing as a test of cure after treatment for cervical intraepithelial neoplasia $(\mathrm{CIN})$ is cost effective

\section{What this study adds}

If realistic assumptions are made about women's compliance with follow-up recommendations, HPV testing is likely to be more effective and cost less than strategies based on annual cytological follow-up of women treated for CIN

\section{Tables}

\begin{tabular}{|c|c|c|}
\hline \multicolumn{3}{|l|}{ Table 1| Costs used in evaluation: 2009 prices } \\
\hline Item & Cost (£) & Source \\
\hline LBC test cost in laboratory* & 7.19 & Moss et $\mathrm{al}^{28}$ \\
\hline HPV reflex test costs* & $12.83 \dagger$ & HPV Sentinel Sites Study \\
\hline $\begin{array}{l}\text { Consultation cost-general practitioner/nurse visit in } \\
\text { community }\end{array}$ & 17.56 & $\begin{array}{l}\text { Includes administration and smear taking time; based on } 80 \% \\
\text { of smears taken by practice nurses }{ }^{25}\end{array}$ \\
\hline Outpatient visit & 71.34 & Martin-Hirsch et al ${ }^{29}$ \\
\hline Colposcopy & 210 & Sherlaw-Johnson and Philips ${ }^{30}$ \\
\hline Punch biopsy & 75.92 & Sherlaw-Johnson and Philips ${ }^{30}$ \\
\hline Cone biopsy & 345.18 & Martin-Hirsch et al ${ }^{29}$ \\
\hline \multicolumn{3}{|l|}{ Treatmentł: } \\
\hline CIN1 & 439.61 & Average cost per event ${ }^{29}$ \\
\hline CIN2/3 & 617.97 & $\begin{array}{l}\text { Average treatment cost of CIN2 and CIN3 from Martin-Hirsch } \\
\text { et } \mathrm{al}^{29}\end{array}$ \\
\hline \multicolumn{3}{|l|}{ Cancer: } \\
\hline Stage I & 2785 & \multirow[t]{4}{*}{ Average cost per event ${ }^{29}$} \\
\hline Stage II & 4448 & \\
\hline Stage III & 12562 & \\
\hline Stage IV & 12777 & \\
\hline
\end{tabular}

$\mathrm{CIN}=$ cervical intraepithelial neoplasia; HPV=human papillomavirus; LBC=liquid based cytology.

*Including storage, transport, laboratory, sample media and vials; this cost is incurred only when cytology result is negative; when cytology result is borderline dyskaryosis or worse, management can be determined on basis of cytology alone.

$\dagger 12.73$ was used in cost effectiveness assessment for HPV used after treatment for CIN.

$\ddagger$ Averaged for proportion receiving loop electrosurgical excision procedure or cone by CIN grade. 
Table 2| Predicted outcomes and resource use over 10 years, per 1000 women treated

\begin{tabular}{|c|c|c|c|}
\hline Recommended strategy & Cytology only follow-up & $\begin{array}{l}\text { HPV test of cure-sentinel sites } \\
\text { protocol }\end{array}$ & $\begin{array}{l}\text { HPV test of cure-extended } \\
\text { follow-up protocol }\end{array}$ \\
\hline \multicolumn{4}{|l|}{ Health outcomes } \\
\hline Residual underlying cases of $\mathrm{CIN} 3+$ at 10 years & 29.1 & 20.7 & 21.5 \\
\hline $\begin{array}{l}\text { Residual underlying cases of CIN3+ averted compared } \\
\text { with current practice }\end{array}$ & - & 8.4 & 7.6 \\
\hline $\begin{array}{l}\text { Cost per additional underlying CIN3+ case averted at } \\
10 \text { years compared with current practice }\end{array}$ & & - 11120 (cost saving) & $£ 6474$ \\
\hline \multicolumn{4}{|l|}{ Resource use } \\
\hline Colposcopies & 406 & 368 & 447 \\
\hline Re-treatments*: & 217 & 272 & 275 \\
\hline At 6 months & 80 & 167 & 167 \\
\hline Between 1 and 10 years & 137 & 104 & 108 \\
\hline Cytology tests & 6197 & 4126 & 5154 \\
\hline HPV tests & - & 1166 & 2035 \\
\hline \multicolumn{4}{|l|}{ Costs } \\
\hline Discounted at $3.5 \%$ per year & $£ 358222$ & $£ 348834$ & $£ 407274$ \\
\hline
\end{tabular}

$\mathrm{CIN}=$ cervical intraepithelial neoplasia; HPV=human papillomavirus.

*Number of re-treatments includes treatment for low grade lesions and cone biopsies done after discordant cytology and colposcopy or unsatisfactory colposcopy after moderate or severe dyskaryosis cytology. 
Table 3| Sensitivity analysis: predicted outcomes and resource use over 10 years, per 1000 women treated if perfect compliance with recommendations is assumed

\begin{tabular}{|c|c|c|c|}
\hline Recommended strategy & Cytology only follow-up & $\begin{array}{l}\text { HPV test of cure-sentinel sites } \\
\text { protocol }\end{array}$ & $\begin{array}{l}\text { HPV test of cure-extended follow-up } \\
\text { protocol }\end{array}$ \\
\hline \multicolumn{4}{|l|}{ Health outcomes } \\
\hline Residual underlying cases of $\mathrm{CIN} 3+$ at 10 years & 6.3 & 11.9 & 11.5 \\
\hline \multirow{2}{*}{$\begin{array}{l}\text { Residual underlying cases of CIN3+ averted } \\
\text { compared with current practice }\end{array}$} & \multirow[t]{2}{*}{-} & -5.6 & -5.1 \\
\hline & & (additional cases) & (additional cases) \\
\hline \multicolumn{4}{|l|}{ Costs } \\
\hline Discounted at $3.5 \%$ per year & $£ 521634$ & $£ 388108$ & $£ 483256$ \\
\hline
\end{tabular}




\section{Figures}

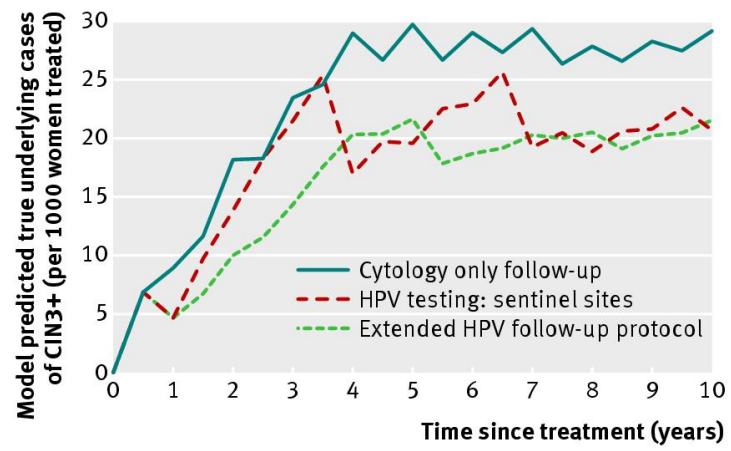



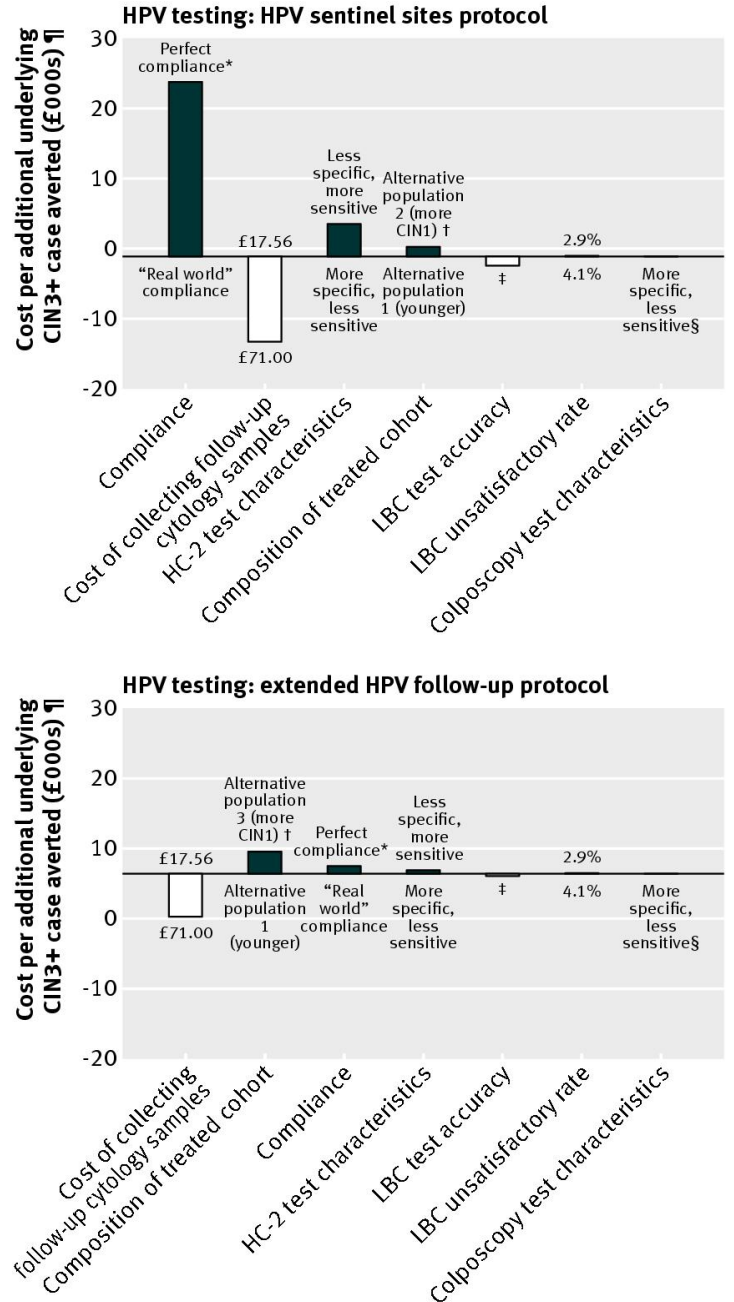

Fig 2 Effect of various model assumptions on cost per additional residual recurrent cervical intraepithelial neoplasia (CIN) 3+ case averted, compared with cytology only follow-up. HPV=human papillomavirus; LBC=liquid based cytology. *Under perfect compliance assumptions, this strategy was predicted to result in more underlying CIN3+ at 10 years than cytology only follow-up, so cost per underlying case of CIN3+ averted should be interpreted as cost per underlying case of CIN3+ averted by cytology only follow-up compared with HPV testing strategy. †Population composition (age structure and proportion treated for CIN1 $v$ CIN2+) was varied to reflect different post-treatment populations observed in HPV Sentinel Sites Study and in a recent study of post-treatment management. ${ }^{2}$ Baseline analyses reflected HPV sentinel sites population, in which $63 \%$ of treated women were younger than 35 years and $10 \%$ of women were treated for CIN1 and the remainder for CIN2+. The "younger" population had a higher proportion of treated women aged $<35$ years $(73 \%)$, consistent with that observed in a previous study ${ }^{2}$; the "more CIN1" population had a higher proportion of women treated for CIN1 (23.6\%), consistent with that observed in a previous study. ${ }^{2} \ddagger$ Sensitivity and specificity varied within feasible range. $\S \mathrm{Effect}$ on outcomes was too small to show substantial variation in this depiction. INegative values indicate that strategy prevents more cases and is cost saving compared with cytology only follow-up 